PROCEEDINGS OF THE

AMERICAN MATHEMATICAL SOCIETY

Volume 133, Number 2, Pages 437-440

S 0002-9939(04)07708-1

Article electronically published on September 2, 2004

\title{
THE OSC DOES NOT IMPLY THE SOSC FOR INFINITE ITERATED FUNCTION SYSTEMS
}

\author{
TOMASZ SZAREK AND STANISŁAW WȨDRYCHOWICZ
}

(Communicated by Michael Handel)

\begin{abstract}
It is shown that every class of contracting similitudes $\left\{f_{1}, \ldots, f_{N}\right\}$ on $R^{s}$ satisfying the OSC and such that $\operatorname{dim}_{H} \mathcal{K}_{0}<s$, where $\mathcal{K}_{0}$ denotes the corresponding fractal, can be extended to an infinite family of contracting similitudes which still satisfies the OSC but the SOSC does not hold.
\end{abstract}

\section{Notation AND DEFinitions}

We consider $R^{s}(s \geq 1)$ with the Euclidean norm $|\cdot|$. By $\operatorname{dist}(A, B)$ we denote the Hausdorff distance of two compact subsets $A, B$ of $R^{s}$. Recall that a map $f: R^{s} \rightarrow$ $R^{s}$ is called a similitude with Lipschitz constant $c \in(0,1)$ if $|f(x)-f(y)|=c|x-y|$ for $x, y \in R^{s}$. Let $I$ be a countable index set with at least two elements, and let $\left\{f_{i}: R^{s} \rightarrow R^{s}: i \in I\right\}$ be a collection of contracting similitudes - a so-called ifs. The class of maps $\left\{f_{i}: i \in I\right\}$ is said to satisfy the Open Set Condition (OSC) if there is a nonempty and bounded open set $U \subset R^{s}$ such that $f_{i}(U) \subset U$ for all $i$, and $f_{i}(U) \cap f_{j}(U)=\emptyset$ for $i \neq j$.

Put $I^{*}=\bigcup_{n \geq 1} I^{n}$ and for $\mathbf{i} \in I^{n}, \mathbf{i}=\left(i_{1}, \ldots, i_{n}\right), n \geq 1$, set

$$
f_{\mathbf{i}}=f_{i_{1}} \circ \ldots \circ f_{i_{n}} .
$$

If $\mathbf{i} \in I^{*} \cup I^{\infty}$ and $n \geq 1$ does not exceed the length of $\mathbf{i}$, we denote by $\mathbf{i}_{n}$ the word $\left(i_{1}, \ldots, i_{n}\right)$. Let $\left\{f_{i}: i \in I\right\}$ satisfy the OSC with a set $U$. Set $X=\operatorname{cl} U$. Observe now that given $\mathbf{i} \in I^{\infty}$, the compact sets $f_{\mathbf{i}_{\mid n}}(X), n \geq 1$, are decreasing and their diameters converge to zero. This implies that the set

$$
\pi(\mathbf{i})=\bigcap_{n=0}^{\infty} f_{\mathbf{i}_{\mid n}}(X)
$$

is a singleton and therefore this formula defines a map $\pi: I^{\infty} \rightarrow X$. The main object of our interest will be the set

$$
\mathcal{K}=\pi\left(I^{\infty}\right)=\bigcup_{\mathbf{i} \in I^{\infty}} \bigcap_{n=0}^{\infty} f_{\mathbf{i}_{\mid n}}(X),
$$

Received by the editors July 2, 2003.

2000 Mathematics Subject Classification. Primary 28A80; Secondary 28A78.

Key words and phrases. Fractal, open set condition, strong open set condition.

The first author's research was supported by the State Committee for Scientific Research Grant No. Z PO3A 03125. 
called the attractor or fractal for $\left\{f_{i}: i \in I\right\}$. We easily check that

$$
\mathcal{K}=\bigcup_{i \in I} f_{i}(\mathcal{K}) .
$$

The Strong Open Set Condition (SOSC) holds if the set $U$ in the definition of the OSC can be chosen in such a way that $\mathcal{K} \cap U \neq \emptyset$.

For every finite word $\mathbf{i}=\left(i_{1}, \ldots, i_{n}\right)$ in the alphabet $I$ we will use the following abbreviation:

$$
c_{\mathbf{i}}=c_{i_{1}} \cdots c_{i_{n}} .
$$

\section{THE THEOREM}

Let $U \subset R^{s}$ be an open set, and let $f_{1}, \ldots, f_{N}$ be given. Assume that $\left\{f_{1}, \ldots, f_{N}\right\}$ satisfies the OSC with the set $U$. A. Schief proved that if $f_{i}, i \in\{1, \ldots, N\}$, are contracting similitudes, then the OSC implies the SOSC (see [6]). Y. Peres et al. showed that the above implication is valid for conformal contractions (see [5]). Recently, infinite families of contracting maps have been extensively studied (see for example 3 ). However, the question (see Problem 7.5 in 3]) of whether the above implication holds in this case seems to be still open. The main aim of our note is to show that for infinite iterated function systems the OSC does not imply the SOSC.

Let $\mathcal{K}_{0}$ denote the attractor for $\left\{f_{1}, \ldots, f_{N}\right\}$. Assume that $\operatorname{dim}_{H} \mathcal{K}_{0}<s$. Hence $\sum_{i=1}^{N} c_{i}^{s}<1$, where $c_{i}$ denotes the Lipschitz constant of $f_{i}, i=1, \ldots, N$ (see [2], [4]). Without loss of generality we may assume that $c_{i} \leq c_{1}$ for all $i \in\{1, \ldots, N\}$.

Let $\left(a_{n}\right)_{n \geq 0}$ be a strictly decreasing sequence of reals such that $1<a_{n} \leq 2$ for all $n \in \mathbb{N}$.

Since $\mathcal{L}^{s}\left(\mathcal{K}_{0}\right)=0$, where $\mathcal{L}^{s}$ is the Lebesgue measure in $R^{s}$, we have $U \backslash \mathcal{K}_{0} \neq$ $\emptyset$. Choose $x \in U \backslash \mathcal{K}_{0}$. Since $x \notin \mathcal{K}_{0}$, there exists $r>0$ such that $f_{j_{1}} \circ \ldots \circ$ $f_{j_{m}}(B(x, 2 r)) \cap B(x, 2 r)=\emptyset$ for $j_{1}, \ldots, j_{m} \in\{1, \ldots, N\}, m \in \mathbb{N}$, and $B(x, 2 r) \subset U$. We may also assume that $\operatorname{dist}\left(B(x, 2 r), \mathcal{K}_{0}\right)>r$.

The following lemma is crucial for our considerations.

Lemma. Let $\left(\left(x_{n}, r_{n}\right)\right)_{n \geq 1}$ be a dense sequence in $\mathrm{cl} U \times(0, r / 2]$, and let $B_{n}=$ $B\left(x_{n}, r_{n}\right)$ for $n \in \mathbb{N}$. Then there are contracting similitudes $f_{N+1}, f_{N+2}, \ldots$ such that there exist sequences $\left(i_{n}\right)_{n \geq 0}$ of integers, $\left(U_{n}\right)_{n \geq 0}$ of open subsets of $U$ and $\left(F_{n}\right)_{n \geq 0}$ of compact subsets of $R^{s}$ satisfying:

(1) $\left(i_{n}\right)_{n \geq 0}$ is nondecreasing, $i_{n} \rightarrow \infty$ as $n \rightarrow \infty$;

(2) $\left\{f_{1}, \ldots, f_{i_{n}}\right\}$ satisfies the $O S C$ with the set $U_{n}$, $\operatorname{dist}\left(B(x, 2 r), \mathcal{K}_{n}\right)>r$ and $\operatorname{dim}_{H} \mathcal{K}_{n}<s$, where $\mathcal{K}_{n}$ denotes the attractor for $\left\{f_{1}, \ldots, f_{i_{n}}\right\}, n \in \mathbb{N} \cup\{0\}$;

(3) $f_{\mathbf{j}}(B(x, 2 r)) \cap B(x, 2 r)=\emptyset$, where $\mathbf{j}$ is a finite word in the alphabet $\left\{1, \ldots, i_{n}\right\}$, $n \in \mathbb{N} \cup\{0\}$;

(4) $B\left(x, a_{n} r\right) \subset U_{n}$ for $n \in \mathbb{N} \cup\{0\}$;

(5) $U_{n}=U_{n-1} \backslash F_{n}, f_{\mathbf{j}}^{-1}\left(F_{n}\right) \cap U_{n-1} \subset F_{n}$, where $\mathbf{j}$ is a finite word in the alphabet $\left\{1, \ldots, i_{n}\right\}, n \in \mathbb{N}$;

(6) $f_{i_{n}}\left(\mathcal{K}_{n-1}\right) \subset B_{n}$ if $B_{n} \cap \mathcal{K}_{n-1} \neq \emptyset$ for $n \in \mathbb{N}$.

Proof. We set $i_{0}=N, U_{0}=U$ and $F_{0}=\emptyset$. Now assume that we are given $i_{p}$, $f_{1}, \ldots, f_{i_{p}}, F_{p}$ and $U_{p}$ for some $p \in \mathbb{N} \cup\{0\}$. We will define $i_{p+1}, f_{i_{p+1}}, F_{p+1}$ and $U_{p+1}$ in such a way that conditions (1)-(6) will be satisfied. If $B_{p+1} \cap \mathcal{K}_{p}=\emptyset$, then we set $i_{p+1}=i_{p}, F_{p+1}=F_{p}$ and $U_{p+1}=U_{p}$. If $B_{p+1} \cap \mathcal{K}_{p} \neq \emptyset$, then $i_{p+1}=i_{p}+1$. Set $r_{0}=\operatorname{dist}\left(B(x, 2 r), \mathcal{K}_{p}\right)$. Obviously $r_{0}>r$. Choose $\eta \in\left(0, r_{0}-r\right)$ 
and set $\varepsilon=\left(1-c_{1}\right) \eta . y \in \operatorname{int}\left(B\left(x, a_{p} r\right) \backslash B\left(x, a_{p+1} r\right)\right)$. Choose $\mathbf{i}$ to be a finite word in the alphabet $\left\{1, \ldots, i_{p}\right\}$ such that $f_{\mathbf{i}}(y) \in B\left(x_{0}, \varepsilon\right)$, and let $\kappa>0$ be such that $B(y, \kappa) \subset \operatorname{int}\left(B\left(x, a_{p} r\right) \backslash B\left(x, a_{p+1} r\right)\right)$ and $f_{\mathbf{i}}(B(y, \kappa)) \subset B\left(x_{0}, \varepsilon\right)$. Since $\mathcal{L}^{s}\left(f_{\mathbf{i}}(B(y, \kappa))\right)>0, \mathcal{L}^{s}\left(\mathcal{K}_{p}\right)=0$ and $\mathcal{K}_{p}$ is compact, there exist $y_{0} \in B(y, \kappa)$ and $\kappa_{0}<\kappa$ such that $\bar{B}\left(y_{0}, \kappa_{0}\right) \subset B(y, \kappa)$ and $f_{\mathbf{i}}\left(\bar{B}\left(y_{0}, \kappa_{0}\right)\right) \cap \mathcal{K}_{p}=\emptyset$, where $\bar{B}\left(y_{0}, \kappa_{0}\right)$ denotes the closed $\kappa_{0}$-ball centered at $y_{0}$. Set $W=f_{\mathbf{i}}\left(\bar{B}\left(y_{0}, \kappa_{0}\right)\right)$. We see at once that

$\#\left\{\mathbf{j}: \mathbf{j}\right.$ is a finite word in the alphabet $\left\{1, \ldots, i_{p}\right\}$ and $\left.f_{\mathbf{j}}^{-1}(W) \cap \operatorname{cl} U \neq \emptyset\right\}<\infty$.

Moreover since $f_{\mathbf{i}}\left(y_{0}\right)$ does not belong to $\mathcal{K}_{p}$, it is not a fixed point for $f_{\mathbf{j}}$, where $\mathbf{j}$ is an arbitrary finite word in the alphabet $\left\{1, \ldots, i_{p}\right\}$. Then, if we choose $\kappa_{0}$ small enough, we can also assume that $W \cap f_{\mathbf{j}}^{-1}(W)=\emptyset$ for all finite words $\mathbf{j}$. Define

$$
F_{p+1}=\bigcup\left(f_{\mathbf{j}}^{-1}(W) \cap \mathrm{cl} U\right),
$$

where the above union is over all finite words in the alphabet $\left\{1, \ldots, i_{p}\right\}$, and observe that by the above $F_{p+1}$ is compact. Further, since $\mathcal{K}_{p}=\operatorname{cl} \bigcup_{\mathbf{j}} \operatorname{Fix}\left(f_{\mathbf{j}}\right)$, where the union is taken over all finite words in the alphabet $\left\{1, \ldots, i_{p}\right\}$ (see [1]), there exists $\mathbf{j}_{0}$ such that $\operatorname{Fix}\left(f_{\mathbf{j}_{0}}\right) \in B\left(x_{0}, \varepsilon\right)$. Without loss of generality we may assume that $\operatorname{diam} f_{\mathbf{j}_{0}}(U)<c_{\mathbf{i}} \kappa_{0}$ and $\sum_{i=1}^{p} c_{i}^{s}+c_{\mathbf{j}_{0}}^{s}<1$. Define $f_{i_{p+1}}=f_{\mathbf{j}_{0}}+\left(f_{\mathbf{i}}\left(y_{0}\right)-\operatorname{Fix}\left(f_{\mathbf{j}_{0}}\right)\right)$ and $U_{p+1}=U_{p} \backslash F_{p+1}$.

We see at once that conditions (1), (3), (5) and (6) hold. Now we check condition (2). From the construction it follows that $f_{i}\left(U_{p+1}\right) \cap f_{j}\left(U_{p+1}\right)=\emptyset$ for $i, j \in$ $\left\{1, \ldots, i_{p+1}\right\}, i \neq j$. To finish the proof of the OSC we have to show that $f_{i}\left(U_{p+1}\right) \subset$ $U_{p+1}$ for $i \in\left\{1, \ldots, i_{p+1}\right\}$. We see at once that $f_{i_{p+1}}\left(U_{p+1}\right) \subset U_{p+1}$. Let $f_{i}(z) \notin$ $U_{p+1}$ for some $z \in U_{n}$ and $i \in\left\{1, \ldots, i_{p+1}-1\right\}$. Then $f_{i}(z) \in F_{p+1}$ and hence $z \in f_{i}^{-1}\left(F_{p+1}\right)$. Further, since $z \in f_{i}^{-1}\left(F_{p+1}\right) \cap U_{p} \subset F_{p+1}$, we obtain $z \notin U_{p+1}=$ $U_{p} \backslash F_{p+1}$, which is a contradiction. From the construction it follows that

$$
\operatorname{dist}\left(\mathcal{K}_{p+1}, \mathcal{K}_{p}\right) \leq \varepsilon\left(1-c_{1}\right)^{-1}
$$

and consequently

$$
\begin{aligned}
\operatorname{dist}\left(\mathcal{K}_{p+1}, B(x, 2 r)\right) & \geq \operatorname{dist}\left(\mathcal{K}_{p+1}, B(x, 2 r)\right)-\operatorname{dist}\left(\mathcal{K}_{p+1}, \mathcal{K}_{p}\right) \\
& \geq \operatorname{dist}\left(\mathcal{K}_{p+1}, B(x, 2 r)\right)-\varepsilon\left(1-c_{1}\right)^{-1} \\
& =\operatorname{dist}\left(\mathcal{K}_{p+1}, B(x, 2 r)\right)-\eta>r,
\end{aligned}
$$

which finishes the proof of (2). To prove (4) assume that $B\left(x, a_{p+1} r\right) \backslash U_{p+1} \neq \emptyset$. We have $B\left(x, a_{p+1} r\right) \subset B\left(x, a_{p} r\right) \subset U_{p}$. Since $U_{p+1}=U_{p} \backslash F_{p+1}$, we have $B\left(x, a_{p+1} r\right) \cap$ $F_{p+1} \neq \emptyset$. By $(3)$ we see that if $f_{\mathbf{i}}\left(B\left(x, a_{p} r\right)\right) \cap f_{\mathbf{j}}\left(B\left(x, a_{p} r\right)\right) \neq \emptyset$, then $\mathbf{i}=\mathbf{j}$. Therefore if $f_{\mathbf{i}}^{-1}(W) \cap B\left(x, a_{p} r\right) \neq \emptyset$ and $f_{\mathbf{j}}^{-1}(W) \cap B\left(x, a_{p} r\right) \neq \emptyset$, we have $\mathbf{i}=\mathbf{j}$. From this it follows that there is a unique finite word $\mathbf{i}$ in the alphabet $\left\{1, \ldots, i_{p+1}\right\}$ such that $f_{\mathbf{i}}^{-1}(W) \subset B\left(x, a_{p} r\right)$. Since $f_{\mathbf{i}}^{-1}(W) \subset B\left(x, a_{p} r\right) \backslash B\left(x, a_{p+1} r\right)$, we have $B\left(x, a_{p+1} r\right) \cap F_{p+1}=\emptyset$, which, in turn, is a contradiction. The proof of (4) is finished.

Theorem. Every class of contracting similitudes $\left\{f_{1}, \ldots, f_{N}\right\}$ on $R^{s}$ satisfying the $O S C$ and such that $\operatorname{dim}_{H} \mathcal{K}_{0}<s$, where $\mathcal{K}_{0}$ denotes the corresponding fractal, can be extended to an infinite family of contracting similitudes which still satisfies the OSC but the SOSC does not hold. 
Proof. We show that an infinite ifs given by the above lemma will satisfy the OSC but will not satisfy the SOSC. Set $U_{0}=U \backslash \mathrm{cl} \bigcup_{i=1}^{\infty} F_{i}$. From conditions (4) and (5) it follows that $U_{0} \neq \emptyset$. Observe that $f_{k}\left(U_{0}\right) \subset U_{0}$ for $k \in \mathbb{N}$. Indeed, for every $k$ there exists $n_{0} \in \mathbb{N}$ such that $i_{n_{0}} \geq k$. Then $f_{k}\left(U_{n_{0}}\right) \subset U_{n_{0}}$. Let $f_{k}(z) \notin U_{0}$ for some $z \in U_{0}$. Therefore there exists a sequence $\left(y_{n}\right)_{n \geq 1}, y_{n} \in \bigcup_{i=1}^{\infty} F_{i}, n \in \mathbb{N}$, such that $y_{n} \rightarrow f_{k}(z) \in U_{n_{0}}=U \backslash \bigcup_{i=1}^{n_{0}} F_{i}$. Hence we may assume that $y_{n} \notin F_{j}$ for $j=1, \ldots, n_{0}$. On the other hand, $f_{k}^{-1}\left(F_{j}\right) \cap U_{0} \subset F_{j}$ for $j>n_{0}$ and

$$
\left|f_{k}^{-1}\left(y_{n}\right)-z\right|=c_{k}^{-1}\left|y_{n}-f_{k}(z)\right| \rightarrow 0
$$

as $n \rightarrow \infty$. Since $f_{k}^{-1}\left(y_{n}\right) \in \bigcup_{i=n_{0}+1}^{\infty} F_{i}$ for all large $n$, we have $z \in \operatorname{cl} \bigcup_{i=n_{0}+1}^{\infty} F_{i} \subset$ $\operatorname{cl} \bigcup_{i=1}^{\infty} F_{i}$, which is a contradiction.

Now we show that $f_{p}\left(U_{0}\right) \cap f_{q}\left(U_{0}\right)=\emptyset$ for $p \neq q$. Namely, choose $m$ such that $i_{m}>\max \{p, q\}$. Then we have $U_{0} \subset U_{m}$ and $\left\{f_{1}, \ldots, f_{i_{m}}\right\}$ satisfies the OSC with $U_{m}$.

Finally, we show that $\left\{f_{1}, f_{2}, \ldots\right\}$ does not satisfy the SOSC. Let $\mathcal{K}$ be its attractor. It is obvious that $\bigcup_{i=1}^{\infty} \mathcal{K}_{i} \subset \mathcal{K} \subset \operatorname{cl} \bigcup_{i=1}^{\infty} \mathcal{K}_{i}$. Suppose to the contrary that the SOSC holds. Then there exist $z \in \mathcal{K}$ and an open set $V$ such that $z \in V$ and $f_{i}(V) \cap f_{j}(V)=\emptyset$ for $i \neq j$. However, since $V$ is open and $z \in \mathcal{K}$, there exists $m \in \mathbb{N}$ such that $\mathcal{K}_{m} \cap V \neq \emptyset$. On the other hand, since $\mathcal{K}_{m}=\operatorname{cl} \bigcup$ Fix $f_{\mathbf{i}}$, where the union is over all finite words $\mathbf{i}$ in the alphabet $\left\{1, \ldots, i_{m}\right\}$ (see [1]), without loss of generality we can assume that $z$ is a fixed point of $f_{\mathbf{i}}$ for some finite word $\mathbf{i}$ in the alphabet $\left\{1, \ldots, i_{m}\right\}$. Let $\tau>0$ be such that $B(z, \tau) \subset V$. Then $f_{\mathbf{i}}(B(z, \tau))=B\left(z, c_{\mathbf{i}} \tau\right)$. Further let $n \in \mathbb{N}$ be such that $i_{n+1} \geq i_{m}$ and $B_{n+1}=B\left(x_{n+1}, r_{n+1}\right)$ satisfies $B_{n+1} \cap \mathcal{K}_{m} \neq \emptyset$ and $B_{n+1} \subset B\left(z, c_{\mathbf{i}} \tau\right)$. Then $B\left(z, c_{\mathbf{i}} \tau\right) \subset f_{\mathbf{i}}(V)$ and $f_{i_{n+1}}\left(\mathcal{K}_{m}\right) \subset B\left(z, c_{\mathbf{i}} \tau\right)$. Hence $f_{\mathbf{i}}(V) \cap f_{i_{n+1}}(V) \neq \emptyset$, which is a contradiction.

\section{ACKNOWLEDGEMENT}

We are grateful to an anonymous referee for remarks and suggestions which have improved the presentation of this paper.

\section{REFERENCES}

[1] Hata M., On the structure of self-similar sets, Japan J. Appl. Math. 2 (1985), 381-414. MR 0839336 (87g:58080)

[2] Hutchinson J., Fractals and self-similarity, Indiana Univ. Math. J. 30 (1981), 713-747. MR 0625600 (82h:49026)

[3] Mauldin R. D. and Urbański M., Dimension and measures in infinite iterated function systems, Proc. London Math. Soc. (3) 73 (1996), 105-154. MR 1387085 (97c:28020)

[4] Moran P. A. P., Additive functions of intervals and Hausdorff measure, Proc. Cambridge Philos. Soc. 42 (1946), 15-23. MR 0014397 (7:278f)

[5] Peres Y., Rams M., Simon K., and Solomyak B., Equivalence of positive Hausdorff measure and the open set condition for self-conformal sets, Proc. Amer. Math. Soc. 129 (2001), 26892699. MR 1838793 (2002d:28004)

[6] Schief A., Separation properties for self-similar sets, Proc. Amer. Math. Soc. 122 (1994), 111-115. MR 1191872 (94k:28012)

Institute of Mathematics, Silesian University - and - Institute of Mathematics of the Polish Academy of Sciences, Bankowa 14, 40-007 Katowice, Poland

E-mail address: szarek@ux2.math.us.edu.p1

Department of Mathematics, Technical University of Rzeszów, W. Pola 6, 35-959 Rzeszów, Poland 